\title{
Advances in the imaging of cerebral aneurysm inflammation
}

\author{
Michael R. Levitt, M. Yashar S. Kalani, Karam Moon, Cameron G. McDougall, Felipe C. Albuquerque \\ Division of Neurological Surgery, Barrow Neurological Institute, St. Joseph's Hospital and Medical Center, \\ Phoenix, AZ 85013, USA.
}

\section{A B S T R A C T}

Cerebral aneurysm formation, growth and rupture are thought to be the result of a complex interaction between cerebrovascular hemodynamics and pathobiology. Recently, new evidence has emerged regarding the role of inflammation in the walls of cerebral aneurysms. Noninvasive methods to characterize the degree of inflammation in aneurysms could enable clinicians to estimate the risk of future aneurysm growth and rupture, influencing treatment. This review examines emerging techniques of imaging inflammatory biomarkers in cerebral aneurysms.

Key words: Ferrosoferric oxide, inflammation, intracranial aneurysm, magnetic resonance angiography, subarachnoid hemorrhage

\section{INTRODUCTION}

Intracranial aneurysms are a substantial source of intracranial hemorrhage worldwide. Many aneurysms are detected incidentally, and the treatment calculus regarding unruptured aneurysms remains debatable. Early studies relied on aneurysm diameter, positing that small aneurysms nearly never ruptured; $;^{[1]}$ however, recent data suggest that some small aneurysms confer a significant rupture risk. ${ }^{[2]}$ More recently, complex morphologic and hemodynamic characteristics have been suggested to risk-stratify unruptured aneurysms for treatment. ${ }^{[3-5]}$ Inflammation is related to hemodynamic stress ${ }^{[6]}$ but relying on only morphologic and hemodynamic factors alone does not account for the role of inflammation in the pathobiology of cerebral aneurysms.

Several studies have demonstrated that inflammation plays a key role in cerebral aneurysm formation and rupture..$^{[7-9]}$ Specifically, the role of macrophages in the response to inflammatory mediators has been proposed as a mechanism for aneurysm rupture. ${ }^{[10,11]}$ However,

\begin{tabular}{|c|c|}
\hline \multicolumn{2}{|c|}{ Access this article online } \\
\hline Quick Response Code: & \multirow[b]{2}{*}{$\begin{array}{l}\text { Website: } \\
\text { www.nnjournal.net }\end{array}$} \\
\hline & \\
\hline & $\begin{array}{l}\text { DOI: } \\
10.4103 / 2347-8659.153970\end{array}$ \\
\hline
\end{tabular}

these studies rely on histological analysis of aneurysm tissue.

Recently, the development of noninvasive imaging of inflammatory markers has been developed and applied to the study of cerebral aneurysms. Preliminary results are promising that the link between aneurysm rupture risk and inflammation is strong, and that such inflammation can be imaged in a clinical setting.

\section{IMAGING OF MYELOPEROXIDASE}

Myeloperoxidase, a potent bactericidal substance primarily housed in the granules of neutrophils, is present in the inflammatory environment. It is present in noninfectious inflammatory reactions such as those accompanying atherosclerosis ${ }^{[9]}$ and vasculopathy. ${ }^{[12]}$

Gounis et al. ${ }^{[13]}$ observed that increased myeloperoxidase expression in aneurysm tissue harvested during surgery was associated with all ruptured aneurysms, as well as those unruptured aneurysms that were considered "high-risk" for rupture based on demographic and anatomic characteristics. The same group has studied a paramagnetic agent (di-5-hydroxytryptamide of gadopentetate dimeglumine) that highlights the presence of myeloperoxidase in animal models of general vascular disease. ${ }^{[14]}$ While no human studies of this magnetic resonance contrast agent have been performed, this represents a promising agent in the noninvasive detection of aneurysmal inflammation.

Corresponding Author: Dr. Felipe C. Albuquerque, C/o Neuroscience Publications, Barrow Neurological Institute, St. Joseph's Hospital and Medical Center, 350 W. Thomas Road, Phoenix, AZ 85013, USA. E-mail: neuropub@dignityhealth.org 


\section{IMAGING OF MACROPHAGE ACTIVITY}

A recent sub-analysis of a large population-based prospective study of unruptured aneurysms demonstrated the protective effect of aspirin on the rupture risk of cerebral aneurysms. ${ }^{[15]}$ The authors posit that the antiinflammatory effects of aspirin confer this risk reduction. In the presence of inflammation, the upregulation of cyclooxygenase-2 (COX-2) and microsomal prostaglandin E2 synthase-1 (mPGES-1) increases prostaglandin production, which in turn increases matrix metalloproteinase-9 (MMP-9) production by macrophages. This causes the degradation of proteins in the extracellular environment. This activity may lead to the weakening of the aneurysm wall, which when exposed to increased hemodynamic stress, leads to aneurysmal rupture. ${ }^{[5,6]}$ Aspirin has been shown to attenuate the expression of COX-2 and mPGES-1, thus reducing the production of MMP-9 by macrophages. ${ }^{[16]}$

Histological studies of ruptured versus unruptured aneurysms have demonstrated an early $(<12 \mathrm{~h})$ macrophage infiltrate, which some authors postulate may be responsible for an acute inflammatory reaction that precipitates aneurysm rupture. ${ }^{[8]}$ The imaging of macrophage activity, therefore, could aid in the detection of a prerupture inflammatory state signaling aneurysm wall instability and urgent need for treatment.

Macrophage imaging has recently been piloted in humans in part due to the development of ferumoxytol. Ferumoxytol is a superparamagnetic form of iron oxide originally developed for the treatment of iron deficiency anemia. ${ }^{[17]}$ Because ferumoxytol is detectable using conventional magnetic resonance imaging (MRI), and cleared by macrophagocytosis, ${ }^{[18]}$ imaging after ferumoxytol infusion can highlight macrophage activity and hence inflammation.
The differential uptake of ferumoxytol infusion can be used to determine the degree of inflammatory activity of macrophages in cerebral vessels [Figure 1]. A histological study of unruptured aneurysms imaged using this modality prior to surgical resection detected iron particles as well as macrophage infiltration in the aneurysm wall, while only macrophages were detected in the tissue of a control group without ferumoxytol infusion. ${ }^{[19]}$

A further study of ferumoxytol correlated its early uptake on serial MRIs with impending aneurysm rupture. ${ }^{[20]}$ Twenty-two patients with thirty unruptured aneurysms underwent MRI after ferumoxytol infusion at 3 time points: immediately, after $24 \mathrm{~h}$, and after $72 \mathrm{~h}$. The presence of ferumoxytol uptake was defined as a reduction in aneurysmal T2 signal when compared to baseline MRI. Ferumoxytol activity was defined as "early" if this change was detected in $24 \mathrm{~h}$, and "late" if detected only $72 \mathrm{~h}$ after infusion. Fourteen aneurysms underwent subsequent surgical clipping, while sixteen were observed based on small aneurysm size, patient age or co-morbidity, or patient preference. Histological analysis was performed on all surgically treated aneurysms, as well as five ruptured aneurysms not imaged using ferumoxytol.

Among surgically repaired aneurysms with early uptake $(n=4)$, immunohistochemical analysis revealed COX-2, mPGES-1 and M1 macrophage activity similar to that of ruptured aneurysms. Those unruptured aneurysms with late uptake $(n=5)$ had significantly less COX-2 and mPGES-1 activity compared to both ruptured aneurysms and unruptured aneurysms with early ferumoxytol uptake.

Of the fourteen aneurysms not surgically treated, three demonstrated early signal changes, eight late

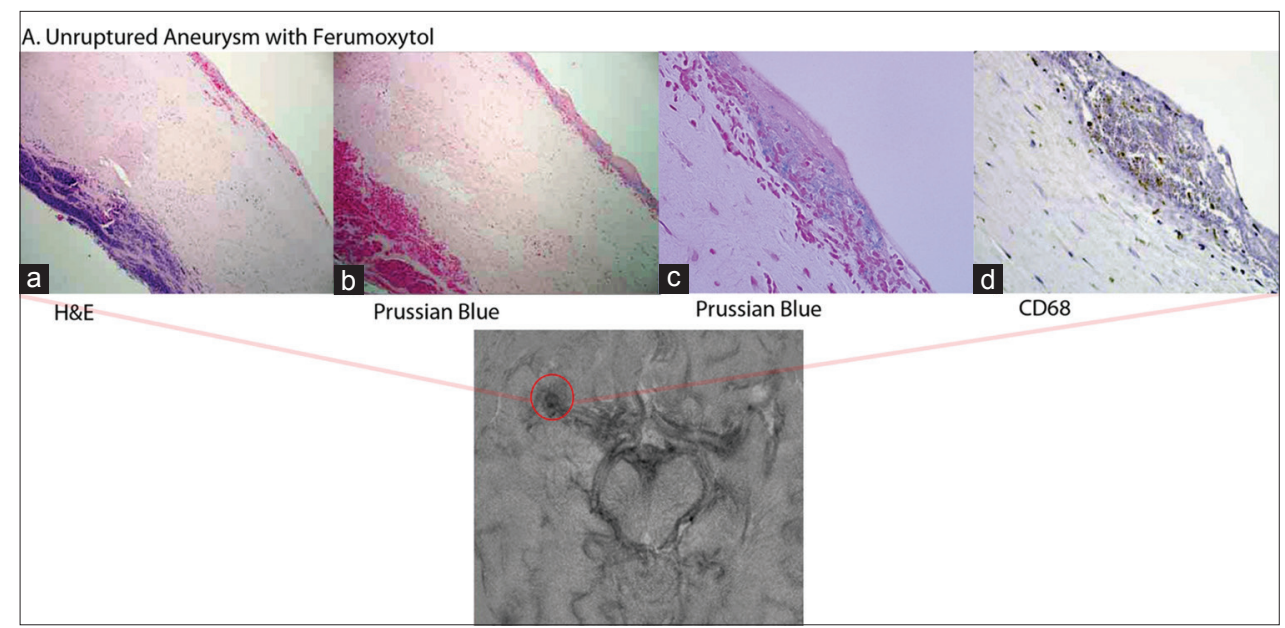

Figure 1: Histology and ferumoxytol-enhanced magnetic resonance imaging (MRI) of an unruptured middle cerebral artery (MCA) aneurysm: (a) HE ×100; (b) Prussian Blue stain showing iron oxide nanoparticles seen mostly in the adventitia; (c) higher magnification of Prussian blue stain demonstrating iron oxide nanoparticles; (d) CD68 showing positive staining for macrophages MRI illustrates right MCA aneurysm with ferumoxytol uptake. Figure adapted with permission from Hasan et al. ${ }^{[19]}$ 
changes, and five no changes. Importantly, all three aneurysms with early signal change ruptured within 6 months of imaging, while none of the remaining aneurysms ruptured or changed in size on follow-up imaging. The authors concluded that aneurysms with early ferumoxytol uptake have a greater degree of inflammation, suggesting an unstable state prone to aneurysm rupture.

The same group then examined the effect of aspirin on ferumoxytol uptake in intracranial aneurysms. A preliminary study demonstrated that administering aspirin $81 \mathrm{mg}$ daily for 3 months to patients with cerebral aneurysms reduced the uptake of ferumoxytol. ${ }^{[21]}$ Subsequently, a small prospective study of eleven patients with twelve unruptured aneurysms compared patients started on daily aspirin $(n=6)$ and a control group $(n=5){ }^{[22]}$ After a baseline ferumoxytol MRI, patients were imaged at 3 months and then underwent surgical aneurysm repair. Patients on aspirin demonstrated an obvious reduction in ferumoxytol uptake compared to baseline, while uptake in the control group did not change. Histological analysis of COX-2, mPGES-1 and macrophages showed significantly lower activity in aneurysms of patients in the aspirin group compared to the control group.

Three conclusions can be drawn from the above ferumoxytol studies. First, ferumoxytol-enhanced MRI can be used to measure aneurysmal inflammation from macrophage activity. Second, early uptake of ferumoxytol is associated with a proinflammatory state that may herald impending aneurysm rupture (though with such a small patient population, definitive statistical conclusions are not possible). Finally, the antiinflammatory effects of aspirin seem to correlate (both radiographically and histologically) with macrophage activity, possibly accounting for the protective effect of aspirin in unruptured aneurysms.

Currently, the role for imaging of aneurysm inflammation is limited to research trials. Future trials of large-scale aneurysm imaging using ferumoxytol are likely the next step in the incorporation of this technique into aneurysm treatment. The limitations of current technology include difficulty interpreting images for evidence of differential uptake of ferumoxytol, which requires experienced neuroradiologists, as well as standardization of the dosage and timing of administration of contrast. In addition, the intriguing finding of reduced inflammation after aspirin administration (and its possible protective effect against aneurysm rupture) is based on a very small number of patients, and further research of a large cohort is required for definitive statistical analysis. Such results could substantially alter the treatment of unruptured aneurysms for which there is treatment equipoise or patient co-morbidity.

\section{CONCLUSION}

Preliminary advances in the imaging of cerebral aneurysm inflammation have highlighted the importance of pathobiology in aneurysm rupture. Future studies of aneurysm surveillance using such imaging, especially ferumoxytol-enhanced MRI, promise to improve clinicians' understanding of individual rupture risk beyond simple anatomical metrics such as aneurysm diameter. Finally, antiinflammatory therapy may play a role in the treatment of unruptured aneurysms.

\section{REFERENCES}

1. Unruptured intracranial aneurysms - risk of rupture and risks of surgical intervention. International study of unruptured intracranial aneurysms investigators. N Engl J Med 1998;339:1725-33.

2. Kashiwazaki D, Kuroda S; Sapporo SAH Study Group. Size ratio can highly predict rupture risk in intracranial small $(<5 \mathrm{~mm})$ aneurysms. Stroke 2013;44:2169-73.

3. Chien A, Sayre J. Morphologic and hemodynamic risk factors in ruptured aneurysms imaged before and after rupture. AJNR Am J Neuroradiol 2014;35:2130-5.

4. Xiang J, Natarajan SK, Tremmel M, Ma D, Mocco J, Hopkins LN, Siddiqui AH, Levy EI, Meng H. Hemodynamic-morphologic discriminants for intracranial aneurysm rupture. Stroke 2011;42:144-52.

5. Meng H, Tutino VM, Xiang J, Siddiqui A. High WSS or low WSS? Complex interactions of hemodynamics with intracranial aneurysm initiation, growth, and rupture: toward a unifying hypothesis. AJNR Am J Neuroradiol 2014;35:1254-62.

6. Turjman AS, Turjman F, Edelman ER. Role of fluid dynamics and inflammation in intracranial aneurysm formation. Circulation 2014;129:373-82.

7. Chyatte D, Bruno G, Desai S, Todor DR. Inflammation and intracranial aneurysms. Neurosurgery 1999;45:1137-46.

8. Frösen J, Piippo A, Paetau A, Kangasniemi M, Niemelä M, Hernesniemi J, Jaaskelainen J. Remodeling of saccular cerebral artery aneurysm wall is associated with rupture: histological analysis of 24 unruptured and 42 ruptured cases. Stroke 2004;35:2287-93.

9. Penn DL, Witte SR, Komotar RJ, Sander Connolly E Jr. The role of vascular remodeling and inflammation in the pathogenesis of intracranial aneurysms. J Clin Neurosci 2014;21:28-32.

10. Hasan D, Hashimoto T, Kung D, Macdonald RL, Winn HR, Heistad D. Upregulation of cyclooxygenase-2 (COX-2) and microsomal prostaglandin E2 synthase-1 (mPGES-1) in wall of ruptured human cerebral aneurysms: preliminary results. Stroke 2012;43:1964-7.

11. Hasan D, Chalouhi N, Jabbour P, Hashimoto T. Macrophage imbalance (M1 vs. M2) and upregulation of mast cells in wall of ruptured human cerebral aneurysms: preliminary results. J Neuroinflammation 2012;9:222.

12. Suzuki K, Okazaki T. Contribution of myeloperoxidase in vasculitis development. Jpn J Infect Dis 2004;57:S2-3.

13. Gounis MJ, Vedantham S, Weaver JP, Puri AS, Brooks CS, Wakhloo AK, Bogdanov AA Jr. Myeloperoxidase in human intracranial aneurysms: preliminary evidence. Stroke 2014;45:1474-7.

14. DeLeo MJ 3rd, Gounis MJ, Hong B, Ford JC, Wakhloo AK, Bogdanov AA Jr. Carotid artery brain aneurysm model: in vivo molecular enzyme-specific MR imaging of active inflammation in a pilot study. Radiology 2009;252:696-703.

15. Hasan DM, Mahaney KB, Brown RD Jr, Meissner I, Piepgras DG, Huston J, Capuano AW, Torner JC. Aspirin as a promising agent 
for decreasing incidence of cerebral aneurysm rupture. Stroke 2011;42:3156-62

16. Xue J, Hua YN, Xie ML, Gu ZL. Aspirin inhibits MMP-9 mRNA expression and release via the PPARalpha/gamma and COX-2/ mPGES-1-mediated pathways in macrophages derived from THP-1 cells. Biomed Pharmacother 2010;64:118-23.

17. Lu M, Cohen MH, Rieves D, Pazdur R. FDA report: ferumoxytol for intravenous iron therapy in adult patients with chronic kidney disease. Am J Hematol 2010;85:315-9.

18. Williams JB, Ye Q, Hitchens TK, Kaufman CL, Ho C. MRI detection of macrophages labeled using micrometer-sized iron oxide particles. J Magn Reson Imaging 2007;25:1210-8.

19. Hasan DM, Mahaney KB, Magnotta VA, Kung DK, Lawton MT, Hashimoto T, Winn HR, Saloner D, Martin A, Gahramanov S, Dosa E, Neuwelt E, Young WL. Macrophage imaging within human cerebral aneurysms wall using ferumoxytol-enhanced MRI: a pilot study. Arterioscler Thromb Vasc Biol 2012;32: 1032-8.

20. Hasan D, Chalouhi N, Jabbour P, Dumont AS, Kung DK, Magnotta VA, Young WL, Hashimoto T, Winn HR, Heistad D. Early change in ferumoxytol-enhanced magnetic resonance imaging signal suggests unstable human cerebral aneurysm: a pilot study. Stroke 2012;43:3258-65.

21. Hasan DM, Chalouhi N, Jabbour P, Magnotta VA, Kung DK, Young WL. Imaging aspirin effect on macrophages in the wall of human cerebral aneurysms using ferumoxytol-enhanced MRI: preliminary results. $J$ Neuroradiol 2013;40:187-91.

22. Hasan DM, Chalouhi N, Jabbour P, Dumont AS, Kung DK, Magnotta VA, Young WL, Hashimoto T, Richard Winn H, Heistad D. Evidence that acetylsalicylic acid attenuates inflammation in the walls of human cerebral aneurysms: preliminary results. J Am Heart Assoc 2013;2:e000019.

Cite this article as: Levitt MR, Kalani MY, Moon K, McDougall CG, Albuquerque FC. Advances in the imaging of cerebral aneurysm inflammation. Neuroimmunol Neuroinflammation 2015;2(2):51-4

Source of Support: Nil. Conflict of Interest: No.

Received: 13-08-2014; Accepted: 01-09-2014 